\title{
Large ameloblastic fibro-odontoma in a 7-year-old girl with analysis of 108 cases
}

\begin{tabular}{l} 
Access this article online \\
\hline Website: \\
www.amsjournal.com \\
\hline DOI: \\
10.4103/2231-0746.186132 \\
\hline Quick Response Code: \\
\hline
\end{tabular}

\author{
Antti Kirjavainen, Veikko Tuovinen, George K. Sándor ${ }^{1,2}$ \\ Department of Oral and Maxillofacial Surgery, Jyväskylä Central Hospital, Jyväskylä, ${ }^{1}$ Department of Oral \\ and Maxillofacial Surgery, Medical Research Center, Oulu University Hospital, Institute of Dentistry, \\ University of Oulu, Oulu, ${ }^{2 B i o M e d i T e c h}$, Institute of Bioscience and Technology, University of \\ Tampere, Tampere, Finland
}

Address for correspondence: Prof. George K. Sándor, University of Oulu, Aapistie 5A, Office No: 2126B, 90014 Oulu, Finland. E-mail: george.sandor@oulu.fi

\section{ABSTRACT}

Background: Odontogenic tumors such as ameloblastic fibro-odontoma (AFO) are rare conditions in children and are often asymptomatic. AFOs are found by routine clinical and radiological examination or when they cause obvious intra- or extra-oral swelling. Materials and Methods: A case of an AFO in a 7-year-old girl is described, and 107 cases from the literature and this report are analyzed. Results: The total of 108 cases revealed the average age at presentation of AFO to be 6.3 years in boys and 9.6 years in girls. There was a slight male predilection and AFO lesions most often occurred in the posterior mandible. AFO was almost always associated with an unerupted tooth or teeth. Conclusions: While the recurrence rate of AFO was found to be $5.5 \%$, long-term postoperative clinical and radiological follow-up is advised to ensure no future signs of aggressive recurrence.

Keywords: Ameloblastic fibro-odontoma, benign tumors, mixed odontogenic tumors

\section{INTRODUCTION}

Ameloblastic fibro-odontoma (AFO) is one of the mixed radiolucent and radiopaque odontogenic tumors. It is relatively rare. Studies indicate that AFO is seen ranging from $0.3 \%$ to $1.7 \%$ of oral pathology biopsy specimens submitted as possible odontogenic tumors. ${ }^{[1,2]}$ AFO was originally termed ameloblastic odontoma before the current nomenclature. Only a few studies exist regarding the characteristics of AFOs as most case reports take the form of single patient publications. ${ }^{[3]}$ This study aimed to review cases of AFO reported consecutively in the literature between the years 1967 and 2015 with the addition of a new case.

\section{Case report}

An otherwise healthy 7-year-old girl was referred by her dentist because of swelling of the left side of the mandible. An asymmetric facial swelling was obvious on extraoral examination [Figure 1]. There was no history of trauma or local infection. Intraoral examination revealed normal mucosa overlying the lesion and the absence of the left mandibular first and second molar teeth [Figure 2]. A distinctive hard bulge was palpable in the vestibule of the left mandible molar region. The mouth opening was normal and there was no pain or other symptoms apart from the swelling. Panoramic radiography [Figure 3] and cone-beam computed tomography scan [Figures 4 and 5] both revealed a large well-defined radiolucent area that contained several radiopaque structures of varying sizes and shapes. The lesion extended from the first molar to the coronoid process and the condylar neck. The borders of the lesion were well circumscribed and the lesion had displaced the developing second molar tooth bud down to the inferior border of the mandible.

This is an open access article distributed under the terms of the Creative Commons Attribution-NonCommercial-ShareAlike 3.0 License, which allows others to remix, tweak, and build upon the work non-commercially, as long as the author is credited and the new creations are licensed under the identical terms. For reprints contact: reprints@medknow.com

Cite this article as: Kirjavainen A, Tuovinen V, Sándor GK. Large ameloblastic fibro-odontoma in a 7-year-old girl with analysis of 108 cases. Ann Maxillofac Surg 2016;6:15-20. 


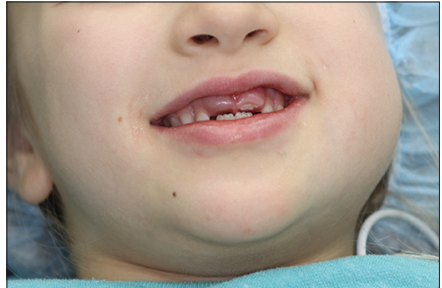

Figure 1: Extraoral swelling of the left side of the mandible

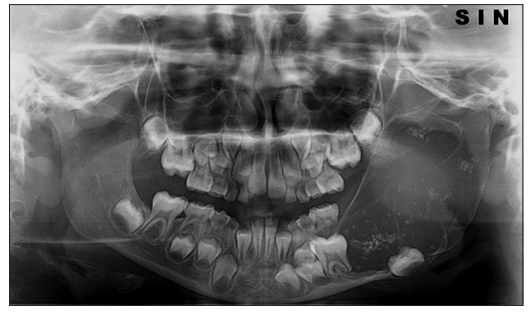

Figure 3: Preoperative panoramic radiograph showing large mixed radiolucent and radiopaque lesion of the left ramus and body of mandible

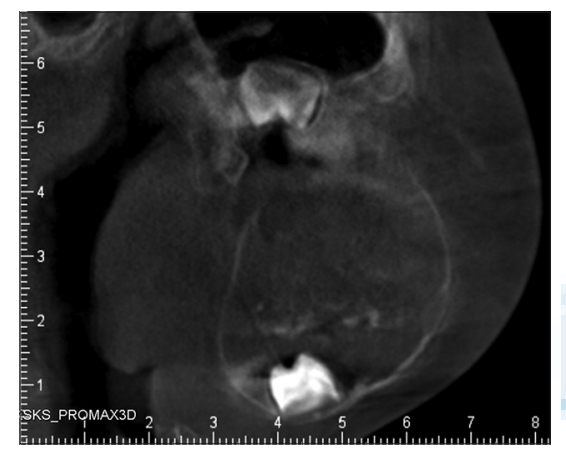

Figure 5: Preoperative cone-beam computed tomography scan coronal view of mixed lesion

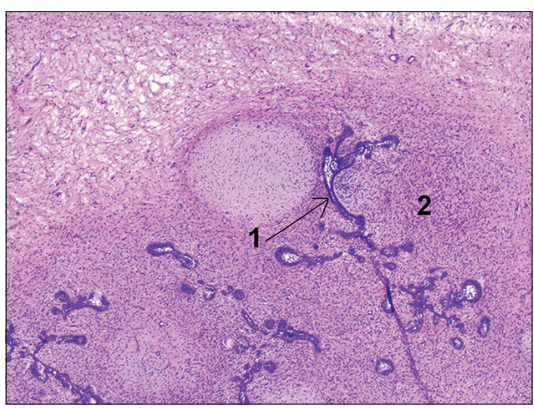

Figure 7: Ameloblastic fibro-odontoma with odontogenic epithelium (1) and odontogenic stroma $(2)(\mathrm{H}$ and $\mathrm{E}, \times 20)$

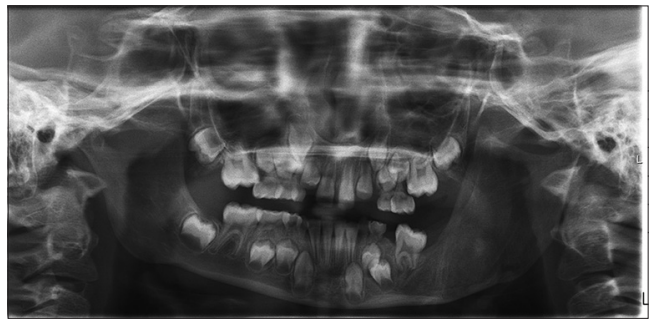

Figure 9: Panoramic radiograph taken 6 months following surgery with healing of intraosseous lesion of the left ramus and body of mandible

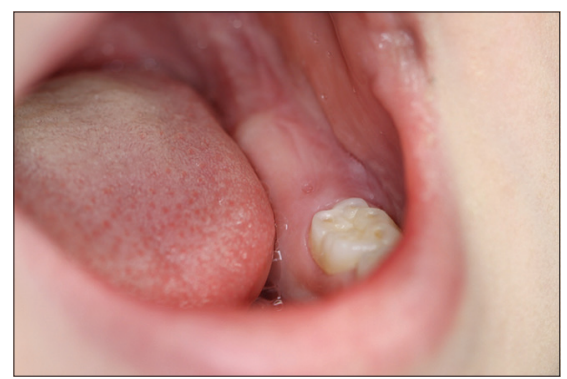

Figure 2: Missing or absent molars of the left side of the mandible

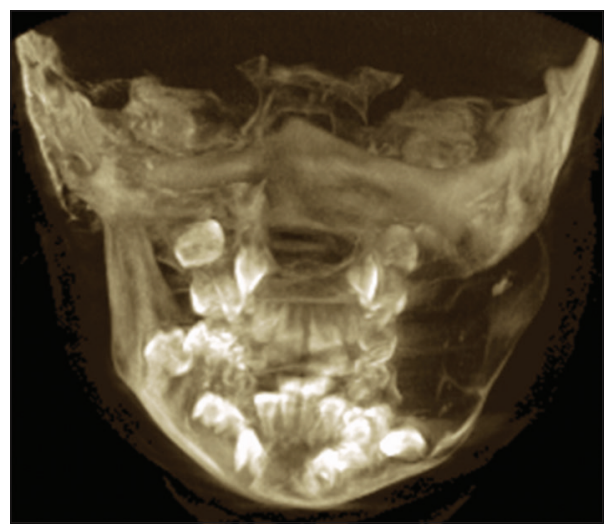

Figure 4: Preoperative cone-beam computed tomography scan revealing large mixed lesion of he left ramus and body of mandible

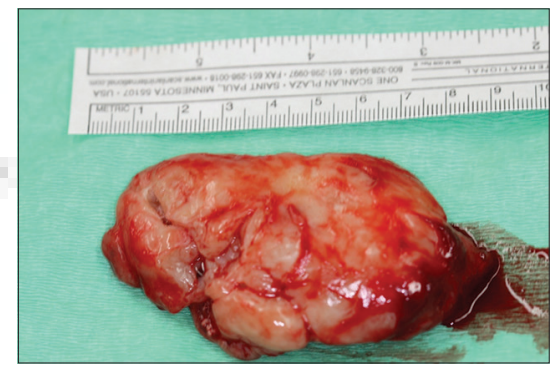

Figure 6: Resected specimen delivered using an intraoral approach

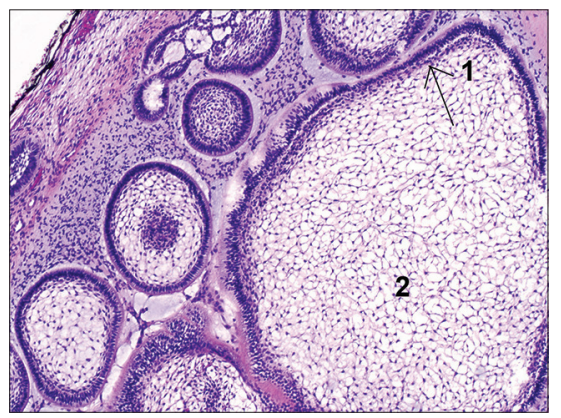

Figure 8: Ameloblastic fibro odontoma exhibiting areas of odontogenic epithelium (1) and (2) (H and E, $\times 40)$

The lesion was enucleated using an intraoral approach under general anesthesia. During the same procedure, tooth numbers 75 and 37 were also extracted. The tumor measured $10 \mathrm{~cm} \times 4 \mathrm{~cm} \times 4 \mathrm{~cm}$ in size [Figure 6] and was submitted for histopathological examination. The samples were stained with hematoxylin and eosin. A large number of epithelial strands and islands of odontogenic epithelium 


\begin{tabular}{|c|c|c|c|c|c|c|c|c|}
\hline First author & $\begin{array}{l}\text { Year of } \\
\text { publication }\end{array}$ & $\begin{array}{l}\text { Number } \\
\text { of cases }\end{array}$ & $\begin{array}{l}\text { Osseous } \\
\text { location }\end{array}$ & Area & $\begin{array}{l}\text { Unerupted } \\
\text { teeth (cases) }\end{array}$ & Sex & $\begin{array}{l}\text { Average } \\
\text { age }\end{array}$ & $\begin{array}{l}\text { Recurrance } \\
\text { reported }\end{array}$ \\
\hline Kirjavainen & 2015 & 1 & Mand $=1$ & Mol & Yes & Female $=1$ & Female $=7$ & No \\
\hline Gantala & $2015^{[2]}$ & 1 & Mand $=1$ & Mol & Yes & Female $=1$ & Female $=11$ & No \\
\hline Surej Kumar & $2014^{[4]}$ & 1 & $\operatorname{Max}=1$ & Mol & Yes & Female $=1$ & Female $=10$ & No \\
\hline Banihashem Rad & $2014^{[5]}$ & 1 & $\operatorname{Max}=1$ & Mol & Yes & Female $=1$ & Female $=11$ & No \\
\hline Lin & $2014^{[6]}$ & 1 & $\operatorname{Max}=1$ & Inc & No & Female $=1$ & Female $=3$ & No \\
\hline Sreenath & $2014^{[7]}$ & 1 & Mand $=1$ & Inc & Yes & Female $=1$ & Female $=5$ & No \\
\hline Nelson & $2014^{[8]}$ & 1 & $\operatorname{Max}=1$ & Mol & Yes & Female $=1$ & Female $=15$ & No \\
\hline Buchner & $2013^{[3]}$ & 10 & $\begin{array}{l}\text { Max }=4 \\
\text { Mand }=6\end{array}$ & $\begin{array}{l}\text { Incisor=1, Premol=1, } \\
\text { Mol=5, Multi=3 }\end{array}$ & $\mathrm{Yes}=9, \mathrm{No}=1$ & $\begin{array}{l}\text { Female }=4, \\
\text { male }=6\end{array}$ & $\begin{array}{l}\text { Female }=14 \\
\text { male }=15\end{array}$ & $U=10$ \\
\hline de Souza Tolentino & $2010^{[9]}$ & 1 & Mand $=1$ & Mol & Yes & Female $=1$ & Female $=11$ & No \\
\hline Wewel & $2010^{[10]}$ & 1 & Mand $=1$ & Inc & Yes & Male $=1$ & Male $=2$ & No \\
\hline Cavalcante & $2009^{[11]}$ & 1 & Mand $=1$ & Mol & Yes & Male $=1$ & Male $=8$ & No \\
\hline De Riu & $2009^{[12]}$ & 1 & $\operatorname{Max}=1$ & Mol & Yes & Male $=1$ & Male $=26$ & No \\
\hline Nascimento & $2009^{[13]}$ & 1 & Mand $=1$ & Multi & Yes & Male $=1$ & Male $=11$ & No \\
\hline Zouhary & $2008^{[14]}$ & 1 & $\operatorname{Max}=1$ & Multi & Yes & Female $=1$ & Female $=7$ & No \\
\hline Hegde & $2008^{[15]}$ & 1 & $\operatorname{Max}=1$ & Multi & Yes & Female $=1$ & Female $=12$ & $U$ \\
\hline Dolanmaz & $2008^{[16]}$ & 1 & $\operatorname{Max}=1$ & Multi & Yes & Female $=1$ & Female $=9$ & No \\
\hline Reis & $2007^{[17]}$ & 1 & Mand $=1$ & Mol & Yes & Female $=1$ & Female $=6$ & No \\
\hline Gyulai-Gaál & $2007^{[18]}$ & 1 & Mand $=1$ & Multi & Yes & Male $=1$ & Male $=9$ & No \\
\hline Chang & $2007^{[19]}$ & 1 & Mand $=1$ & Mol & Yes & Female $=1$ & Female $=16$ & $U$ \\
\hline Oghli & $2007^{[20]}$ & 1 & Mand $=1$ & Multi & Yes & Male $=1$ & Male $=3,5$ & No \\
\hline Silva & $2006^{[21]}$ & 1 & Mand $=1$ & Mol & Yes & Male $=1$ & Male $=14$ & No \\
\hline $\mathrm{Hu}$ & $2006^{[22]}$ & 1 & $\operatorname{Max}=1$ & Multi & Yes & Female $=1$ & Female $=17$ & No \\
\hline Soares & $2006^{[23]}$ & 1 & $\operatorname{Max}=1$ & Multi & Yes & Male $=1$ & Male $=4$ & $\mathrm{U}$ \\
\hline Chen & $2005^{[24]}$ & 7 & $\begin{array}{l}\text { Max }=2, \\
\text { Mand }=5\end{array}$ & $\mathrm{Inc}=1, \mathrm{Mol}=4$, Multi $=2$ & Yes & $\begin{array}{l}\text { Female }=3, \\
\text { male }=4\end{array}$ & $\begin{array}{l}\text { Male }=10 \\
\text { female }=10\end{array}$ & $\begin{array}{l}Y e s=3, n o=2, \\
U=2\end{array}$ \\
\hline Sivapathasundharam & $2005^{[25]}$ & 1 & Mand $=1$ & Multi & Yes & Male $=1$ & Male $=17$ & $\mathrm{U}$ \\
\hline Reichart & $2004^{[26]}$ & 2 & Mand $=2$ & $\mathrm{Mol}=2$ & Yes, No & Male $=2$ & Male $=10$ & $\mathrm{No}=1, \mathrm{U}=1$ \\
\hline Dhanuthai & $2004^{[27]}$ & 1 & $\operatorname{Max}=1$ & Inc & Yes & Female $=1$ & Female $=1$ & No \\
\hline Alderson & $2004^{[28]}$ & 1 & Mand $=1$ & Mol & Yes & Male $=1$ & Male $=11$ & U \\
\hline Chang & $2002^{[29]}$ & 1 & Mand $=1$ & Mol & Yes & Female $=1$ & Female $=26$ & No \\
\hline Mosqueda-Taylor & $2002^{[30]}$ & 3 & $\begin{array}{l}\text { Max }=2, \\
\text { Mand }=1\end{array}$ & Multi $=3$ & Yes & $\begin{array}{l}\text { Female }=1, \\
\text { male }=2\end{array}$ & $\begin{array}{l}\text { Female }=25, \\
\text { male }=12\end{array}$ & $\mathrm{U}=1, \mathrm{No}=2$ \\
\hline Friedrich & $2001^{[31]}$ & 1 & Mand $=1$ & Mol & Yes & Male $=1$ & Male $=8$ & U \\
\hline Yagishita & $2001^{[32]}$ & 1 & Mand $=1$ & Multi & No & Male $=1$ & Male $=24$ & U \\
\hline al-Sebaei & $2001^{[33]}$ & 1 & $\operatorname{Max}=1$ & Multi & Yes & Male $=1$ & Male $=11$ & U \\
\hline Flaitz & $2001^{[34]}$ & 1 & Mand $=1$ & Inc & Yes & Male $=1$ & Male $=2,5$ & U \\
\hline Steinberg & $2001^{[35]}$ & 1 & $\operatorname{Max}=1$ & Multi & Yes & Male $=1$ & Male $=6$ & U \\
\hline Rao & $1999^{[36]}$ & 2 & Mand $=2$ & Multi & Yes & Male $=2$ & Male $=10,5$ & $\mathrm{U}=2$ \\
\hline Furst & $1999^{[37]}$ & 1 & Mand $=1$ & Mol & Yes & Male $=1$ & Male $=7$ & Yes \\
\hline Savitha & $1998^{[38]}$ & 1 & Mand $=1$ & Multi & Yes & Male $=1$ & Male $=5$ & No \\
\hline Favia & $1997^{[39]}$ & 2 & $\begin{array}{l}\text { Max }=1 \\
\text { Mand }=1\end{array}$ & Multi $=2$ & U & Male $=2$ & Male $=3,5$ & $\mathrm{No}=2$ \\
\hline Ozer & $1997^{[40]}$ & 1 & $\operatorname{Max}=1$ & Multi & Yes & Female $=1$ & Female $=7$ & No \\
\hline Kitano & $1994^{[41]}$ & 1 & Mand $=1$ & Mol & Yes & Female $=1$ & Female $=9$ & No \\
\hline Baker & $1993^{[42]}$ & 1 & $\operatorname{Max}=1$ & Inc & Yes & Male $=1$ & Male $=0,75$ & No \\
\hline Okura & $1992^{[43]}$ & 2 & $\begin{array}{l}\text { Mand =1, } \\
\operatorname{Max}=1\end{array}$ & $\mathrm{Mol}=2$ & Yes & $\begin{array}{l}\text { Male }=1 \\
\text { female }=1\end{array}$ & $\begin{array}{l}\text { Male }=7 \\
\text { female }=12\end{array}$ & $\mathrm{No}=2$ \\
\hline Piette & $1990^{[44]}$ & 1 & $\operatorname{Max}=1$ & Multi & Yes & Male $=1$ & Male $=7$ & U \\
\hline Glickman & $1989^{[45]}$ & 1 & Mand $=1$ & Mol & Yes & Female $=1$ & Female $=15$ & U \\
\hline Warnock & $1989^{[46]}$ & 1 & Mand $=1$ & Multi & Yes & Male $=1$ & Male $=11$ & U \\
\hline Hansen & $1988^{[47]}$ & 8 & $\begin{array}{l}\text { Mand }=4, \\
\operatorname{Max}=4\end{array}$ & $U=8$ & $\mathrm{U}$ & $\begin{array}{l}\text { Male }=6 \\
\text { female }=2\end{array}$ & $\begin{array}{l}\text { Male }=11,5, \\
\text { female }=8\end{array}$ & $\mathrm{No}=4, \mathrm{U}=4$ \\
\hline Takeda & $1988^{[48]}$ & 1 & $\mathrm{Max}=1$ & Mol & Yes & Female $=1$ & Female $=11$ & No \\
\hline Hawkins & $1986^{[49]}$ & 1 & $\operatorname{Max}=1$ & Mol & Yes & Female $=1$ & Female $=6,5$ & U \\
\hline Sole & $1986^{[50]}$ & 1 & Mand $=1$ & Mol & Yes & Male $=1$ & Male $=12$ & U \\
\hline Reich & $1984^{[51]}$ & 1 & Mand $=1$ & Mol & Yes & Female $=1$ & Female $=11$ & No \\
\hline Anneroth & $1982^{[52]}$ & 1 & $\operatorname{Max}=1$ & Inc & Yes & Male $=1$ & Male $=7$ & No \\
\hline Hutt & $1982^{[53]}$ & 1 & Mand $=1$ & Mol & Yes & Female $=1$ & Female $=11$ & No \\
\hline Daley & $1982^{[54]}$ & 1 & $\operatorname{Max}=1$ & Mol & Yes & Female $=1$ & Female $=4$ & U \\
\hline Curran & $1980^{[55]}$ & 1 & Mand $=1$ & Multi & Yes & Male $=1$ & Male $=15$ & No \\
\hline Bernhoft & $1979^{[56]}$ & 1 & $\operatorname{Max}=1$ & Mol & Yes & Female $=1$ & Female $=14$ & No \\
\hline Geller & $1978^{[57]}$ & 1 & $\operatorname{Max}=1$ & Mol & Yes & Male $=1$ & Female $=15$ & No \\
\hline Howell & $1977^{[58]}$ & 2 & Mand $=2$ & Multi $=2$ & $\mathrm{U}$ & $\begin{array}{l}\text { Male }=1 \\
\text { female }=1\end{array}$ & $\begin{array}{l}\text { Male }=36 \\
\text { female }=18\end{array}$ & $\mathrm{Yes}=2$ \\
\hline Album & $1977^{[59]}$ & 1 & Mand $=1$ & Multi & Yes & Male=1 & Male $=5$ & No \\
\hline
\end{tabular}




\begin{tabular}{|c|c|c|c|c|c|c|c|c|}
\hline First author & $\begin{array}{l}\text { Year of } \\
\text { publication }\end{array}$ & $\begin{array}{l}\text { Number } \\
\text { of cases }\end{array}$ & $\begin{array}{l}\text { Osseous } \\
\text { location }\end{array}$ & Area & $\begin{array}{l}\text { Unerupted } \\
\text { teeth (cases) }\end{array}$ & Sex & $\begin{array}{l}\text { Average } \\
\text { age }\end{array}$ & $\begin{array}{l}\text { Recurrance } \\
\text { reported }\end{array}$ \\
\hline Herd & $1977^{[60]}$ & 1 & Mand $=1$ & Mol & Yes & Female $=1$ & Female $=11$ & No \\
\hline Eda & $1977^{[61]}$ & 1 & $\operatorname{Max}=1$ & Multi & Yes & Female $=1$ & Female $=22$ & No \\
\hline Cran & $1976^{[62]}$ & 1 & Mand $=1$ & Mol & Yes & Male $=1$ & Male $=9$ & U \\
\hline Hanna & $1976^{[63]}$ & 1 & Mand =1 & Multi & Yes & Male $=1$ & Male $=11$ & No \\
\hline Miller & $1976^{[64]}$ & 7 & $\begin{array}{l}\text { Mand }=4 \\
\operatorname{Max}=3\end{array}$ & Mol=4, Multi $=3$ & $\mathrm{Yes}=4, \mathrm{U}=3$ & $\begin{array}{l}\text { Male }=5 \\
\text { female }=2\end{array}$ & $\begin{array}{l}\text { Male }=11 \\
\text { female }=12\end{array}$ & $\mathrm{No}=7$ \\
\hline Meerkotter & $1974^{[65]}$ & 1 & Mand=1 & Mol & Yes & Male $=1$ & Male $=9$ & No \\
\hline Sanders & $1974^{[66]}$ & 1 & Mand $=1$ & Mol & Yes & Male $=1$ & Male $=19$ & No \\
\hline Worley & $1972^{[67]}$ & 1 & Mand $=1$ & Multi & Yes & Male $=1$ & Male $=11$ & $\mathrm{U}$ \\
\hline $0 `$ Brien & $1971^{[68]}$ & 1 & $\operatorname{Max}=1$ & U & $U$ & Female $=1$ & Female $=2,5$ & $\mathrm{U}$ \\
\hline Dutta & $1970^{[69]}$ & 1 & $\operatorname{Max}=1$ & Multi & Yes & Male $=1$ & Male $=8$ & No \\
\hline Jacobsohn & $1968^{[70]}$ & 1 & Mand $=1$ & Premol & No & Male $=1$ & Male $=15$ & No \\
\hline Hamner & $1968^{[71]}$ & 2 & Mand $=2$ & $\mathrm{Mol}=2$ & Yes $=2$ & Male $=2$ & Male $=7$ & $\mathrm{No}=2$ \\
\hline Olech & $1967^{[72]}$ & 1 & $\operatorname{Max}=1$ & Inc & Yes & Female $=1$ & Female $=5$ & No \\
\hline Total & & 108 & $\begin{array}{l}\text { Max }=43 \\
\text { Mand }=65\end{array}$ & $\begin{array}{l}\text { Inc }=10, \text { Premol=2, } \\
\text { Mol= } 49, \text { Multi }=38, U=9\end{array}$ & $\begin{array}{l}\text { Yes }=96 \\
\text { no }=5, U=7\end{array}$ & $\begin{array}{l}\text { Male }=66 \\
\text { female }=42\end{array}$ & $\begin{array}{l}\text { Female }=9,6 \\
\text { male }=6,3\end{array}$ & $\begin{array}{l}\text { No }=62 \\
\text { Yes }=6, U=40\end{array}$ \\
\hline
\end{tabular}

Max=Maxilla; Mand=Mandible; Inc=Incisor; Premol=Premolar; Mol=Molar; Multi=Multiple sites; $U=$ Unknown. Numerical superscript next to year of publication indicates citation number of report in references section of manuscript

\begin{tabular}{ll}
$\begin{array}{l}\text { Table 2: Characteristics of } 108 \text { ameloblastic } \\
\text { fibro-odontoma lesions }\end{array}$ \\
\hline Characteristic & Ratio \\
\hline Female to male ratio & $1: 1,65$ \\
Average age (years) & Female: 9.6 \\
& Male: 6.3 \\
Mandible versus maxilla (\%) & $65(59.43)$ versus 43 (40.57) \\
Molar region (\%) & Molar: 47 (44.34) \\
& Molar + multiple sites: $85(80.19)$ \\
Unerupted teeth (\%) & $94(88.68)$ \\
Recurrence (\%) & $6(5.57)$ \\
\hline
\end{tabular}

were found. The hard tissue component consisted of dentin and enamel. Histopathological examination [Figures 7 and 8] confirmed the clinical and radiological diagnosis of AFO. Clinical and radiological follow-up [Figure 9] was continued over 24 months and the postoperative course was uneventful with no recurrences to date.

\section{MATERIALS AND METHODS}

A search was conducted using Medline and Scopus databases using the search words AFO and odontogenic tumors. This yielded a total of 1254 hits which included many lesions other than AFO, such as ameloblastic fibroma (AF), ameloblastic fibrodentinoma, ameloblastic fibrosarcoma, and odontoma. The search was restricted to AFO and reports including the other diagnoses were removed. The criteria for inclusion in this review were reports describing radiographic features consistent with $\mathrm{AFO}$, histologic features consistent with AFO, and minimum follow-up of 6 months following removal of the lesion. Any report or study failing to mention any of the above inclusion criteria was excluded. This yielded a total of 107 cases which together with the newly reported case of the 7-year-old girl comprising 108 cases forming the database of this review.

\section{RESULTS}

A summary of the findings of AFO reports is presented in Table 1. AFO was found to be a benign tumor of children with the average age at presentation being 6.3 years in males and 9.6 years in females. AFO had a female: male ratio of 1:1.62. Typically, AFO lesions were located in the posterior region of the mandible in $60 \%$ of cases. Almost all lesions, 94 of $106(89 \%)$, were associated with the crown of an unerupted tooth or teeth. Clinically, AFO was usually painless and was characterized by asymmetric swelling of the face and delayed tooth eruption. Radiological features of AFO included a cyst-like lesion with radiopaque areas resembling a developing odontoma. The recurrence rate in this review of 108 cases was only 6 recurrent cases or $5.5 \%$ [Tables 1 and 2].

\section{DISCUSSION}

In defining AFO, the World Health Organization lists it as a rare odontogenic tumor with the histologic features of $\mathrm{AF}$ in conjunction with the presence of dentin and enamel. ${ }^{[73]}$ Histopathologically, the tumor is composed of both soft and hard tissues. The soft tissue component is composed of epithelial strands and small islands of odontogenic epithelium associated with a primitive appearing myxoid connective tissue which resembles the dental papilla [Figures 7 and 8]. The hard tissue component consists of foci of enamel and dentin. ${ }^{[74]}$

Studies have shown that AFO is usually not aggressive and can be treated surgically without removal of the adjacent teeth. ${ }^{[43,68,71,70,75]}$ A few studies suggest that AFO can behave aggressively with multiple recurrences. ${ }^{[2,76]}$ There had been suggestions that a primarily benign AFO can undergo malignant transformation to ameloblastic fibrosarcoma. ${ }^{[58,77-79]}$ Therefore, it is important to continue the follow-up of AFO lesions for several years.

\section{CONCLUSIONS}

AFO is a rare benign mixed odontogenic tumor. Normally, AFO is found at the average age of 9 years in females and 6 years in males. The prognosis is good. The correct diagnosis is based on clinical, radiological, and histopathological findings. AF and complex odontoma must be considered in the differential 
diagnosis. The treatment of choice is to remove tumor surgically usually by enucleation.

In this newly reported case, the AFO tumor was enucleated and the area of the left posterior mandible healed uneventfully without complications. Clinical and radiological follow-up has continued for 24 months with no signs of recurrence. Follow-up must be continued on a regular basis for several years. The patient in this case report is now receiving orthodontic treatment and remains under vigilant surgical follow-up.

\section{Financial support and sponsorship}

Nil.

\section{Conflicts of interest}

There are no conflicts of interest.

\section{REFERENCES}

1. Buchner A, Kaffe I, Vered M. Clinical and radiological profile of ameloblastic fibro-odontoma: An update on an uncommon odontogenic tumor based on a critical analysis of 114 cases. Head Neck Pathol 2013;7:54-63.

2. Gantala R, Gotoor SG, Kumar RV, Munisekhar MS. Ameloblastic fibro-odontoma. BMJ Case Rep 2015;2015. pii: Bcr2015209739.

3. Buchner A, Vered M. Ameloblastic fibroma: A stage in the development of a hamartomatous odontoma or a true neoplasm? Critical analysis of 162 previously reported cases plus 10 new cases. Oral Surg Oral Med Oral Pathol Oral Radiol 2013;116:598-606.

4. Surej Kumar LK, Manuel S, Khalam SA, Venugopal K, Sivakumar TT, Issac J. Ameloblastic fibro-odontoma. Int J Surg Case Rep 2014;5:1142-4.

5. Banihashem Rad SA, Mortazavi H, Eshghpour M, Salehinejad J, Shahakbari R. A large ameloblastic fibro-odontoma of the maxillary sinus. Iran J Otorhinolaryngol 2014;26:111-4.

6. Lin YC, Hsu HM, Liu CS, Yuan K. A peripheral ameloblastic fibro-odontoma in a 3-year-old girl: Case report, immunohistochemical analysis, and literature review. Case Rep Dent 2014;2014:321671.

7. Sreenath G, Indira Reddy Y, Sreenivasreddy P. Ameloblastic fibro-odontoma (AFO) of the mandible: A case report. J Clin Diagn Res 2014;8:260-2.

8. Nelson BL, Thompson LD. Ameloblastic fibro-odontoma. Head Neck Pathol 2014;8:168-70.

9. de Souza Tolentino E, Centurion BS, Lima MC, Freitas-Faria P, Consolaro A, Sant'ana E. Ameloblastic fibro-odontoma: A diagnostic challenge. Int J Dent 2010;2010. pii: 104630.

10. Wewel J, Narayana N. Ameloblastic fibro-odontoma of the anterior mandible in a 22-month-old boy. Indian J Dent Res 2010;21:618-20.

11. Cavalcante AS, Anbinder AL, Costa NC, Lima JR, Carvalho YR. Ameloblastic fibro-odontoma: A case report. Med Oral Patol Oral Cir Bucal 2009;14:e650-3.

12. De Riu G, Meloni SM, Contini M, Tullio A. Ameloblastic fibro-odontoma. Case report and review of the literature. J Craniomaxillofac Surg 2010;38:141-4

13. Nascimento JE, Araújo LD, Almeida LY, De Paula AM, Bonan PR. Ameloblastic fibro-odontoma: A conservative surgical approach. Med Oral Patol Oral Cir Bucal 2009;14:e654-7.

14. Zouhary KJ, Said-Al-Naief N, Waite PD. Ameloblastic fibro-odontoma: Expansile mixed radiolucent lesion in the posterior maxilla: A case report. Oral Surg Oral Med Oral Pathol Oral Radiol Endod 2008;106:e15-21.

15. Hegde V, Hemavathy S. A massive ameloblastic fibro-odontoma of the maxilla. Indian J Dent Res 2008;19:162-4.

16. Dolanmaz D, Pampu AA, Kalayci A, Etöz OA, Atici S. An unusual size of ameloblastic fibro-odontoma. Dentomaxillofac Radiol 2008;37:179-82.

17. Reis SR, de Freitas CE, do Espírito Santo AR. Management of ameloblastic fibro-odontoma in a 6-year-old girl preserving the associated impacted permanent tooth. J Oral Sci 2007;49:331-5.

18. Gyulai-Gaál S, Takács D, Szabó G, Suba Z. Mixed odontogenic tumors in children and adolescents. J Craniofac Surg 2007;18:1338-42.

19. Chang JY, Marcantoni H, Kessler HP. Oral and maxillofacial pathology case of the month. Ameloblastic fibro-odontoma. Tex Dent J 2007;124:686-7, 694-5.

20. Oghli AA, Scuto I, Ziegler C, Flechtenmacher C, Hofele C. A large ameloblastic fibro-odontoma of the right mandible. Med Oral Patol Oral Cir Bucal 2007;12:E34-7.

21. Silva GC, Jham BC, Silva EC. Ameloblastic fibro-odontoma. Oral Oncol Extra 2006;42:217-20.

22. Hu Y, Liu B, Su T. A huge ameloblastic fibro-odontoma of the maxilla. Oral Oncol Extra 2006;42:160-2.

23. Soares RC, Godoy GP, Neto JC. Ameloblastic fibro-odontoma: Report of a case presenting an unusual clinical course. Int J Pediatr Otorhinolaryngol 2006; 1:200-3.

24. Chen Y, Li TJ, Gao Y, Yu SF. Ameloblastic fibroma and related lesions: A clinicopathologic study with reference to their nature and interrelationship. J Oral Pathol Med 2005;34:588-95.

25. Sivapathasundharam B, Manikandhan R, Sivakumar G, George T. Ameloblastic fibro odontoma. Indian J Dent Res 2005;16:19-21.

26. Reichart PA, Philipsen HP, Gelderblom HR. Ameloblastic fibro-odontoma: Report of two cases with ultrastructural study of tumour dental hard structures. Oral Oncol 2004;40:8-12.

27. Dhanuthai K, Kongin K. Ameloblastic fibro-odontoma: A case report. J Clin Pediatr Dent 2004;29:75-7.

28. Alderson GL, McGuff HS, Jones AC, Bandy DP. Oral and maxillofacial pathology case of the month. Ameloblastic fibro-odontoma. Tex Dent J 2004;121:427, 431.

29. Chang H, Precious DS, Shimizu MS. Ameloblastic fibro-odontoma: A case report. J Can Dent Assoc 2002;68:243-6.

30. Mosqueda-Taylor A, Carlos-Bregni R, Ramírez-Amador V, Palma-Guzmán JM, Esquivel-Bonilla D, Hernández-Rojase LA. Odontoameloblastoma. Clinico-pathologic study of three cases and critical review of the literature. Oral Oncol 2002;38:800-5.

31. Friedrich RE, Siegert J, Donath K, Jäkel KT. Recurrent ameloblastic fibro-odontoma in a 10-year-old boy. J Oral Maxillofac Surg 2001;59:1362-6.

32. Yagishita $H$, Taya $Y$, Kanri $Y$, Matsuo A, Nonaka H, Fujita $H$, et al. The secretion of amelogenins is associated with the induction of enamel and dentinoid in an ameloblastic fibro-odontoma. J Oral Pathol Med 2001;30:499-503.

33. 33al-Sebaei MO, Gagari E. Ameloblastic fibro-odontoma. J Mass Dent Soc 2001;50:52-3.

34. Flaitz CM, Hicks J. Delayed tooth eruption associated with an ameloblastic fibro-odontoma. Pediatr Dent 2001;23:253-4.

35. Steinberg MJ, Herrera AF, Frontera Y. Mixed radiographic lesion in the anterior maxilla in a 6-year-old boy. J Oral Maxillofac Surg 2001;59:317-21.

36. Rao NR. Ameloblastic fibro-odontoma: Report of two cases. J Indian Dent Assoc 1999;70:90-1.

37. Furst I, Pharoah M, Phillips J. Recurrence of an ameloblastic fibro-odontoma in a 9-year-old boy. J Oral Maxillofac Surg 1999;57:620-3.

38. Savitha K, Cariappa KM. An effective extraoral approach to the mandible. A technical note. Int J Oral Maxillofac Surg 1998;27:61-2.

39. Favia GF, Di Alberti L, Scarano A, Piattelli A. Ameloblastic fibro-odontoma: Report of two cases. Oral Oncol 1997;33:444-6.

40. Ozer E, Pabuccuoglu U, Gunbay U, Sarioglu S, Aktas S. Ameloblastic fibro-odontoma of the maxilla: Case report. J Clin Pediatr Dent 1997;21:329-31.

41. Kitano M, Tsuda-Yamada S, Semba I, Mimura T, Nozoe E, Setoyama M. Pigmented ameloblastic fibro-odontoma with melanophages. Oral Surg Oral Med Oral Pathol 1994;77:271-5.

42. Baker WR, Swift JQ. Ameloblastic fibro-odontoma of the anterior maxilla. Report of a case. Oral Surg Oral Med Oral Pathol 1993;76:294-7.

43. Okura M, Nakahara H, Matsuya T. Treatment of ameloblastic fibro-odontoma without removal of the associated impacted permanent tooth: Report of cases. J Oral Maxillofac Surg 1992;50:1094-7.

44. Piette EM, Tideman H, Wu PC. Massive maxillary ameloblastic fibro-odontoma: Case report with surgical management. J Oral Maxillofac Surg 1990;48:526-30.

45. Glickman R, Salman L, Chaudhry AP. Ameloblastic fibro-odontoma: A case report. Ann Dent 1989;48:25-7. 
46. Warnock GR, Pankey G, Foss R. Well-circumscribed mixed-density lesion coronal to an unerupted permanent tooth. J Am Dent Assoc 1989;119:311-2.

47. Hansen LS, Ficarra G. Mixed odontogenic tumors: An analysis of 23 new cases. Head Neck Surg 1988;10:330-43.

48. Takeda Y, Suzuki A, Kuroda M, Itagaki M, Shimono M. Pigmented ameloblastic fibro-odontoma: Detection of melanin pigment in enamel. Bull Tokyo Dent Coll 1988;29:119-23.

49. Hawkins PL, Sadeghi EM. Ameloblastic fibro-odontoma: Report of case. J Oral Maxillofac Surg 1986;44:1014-9.

50. Sole MS, Jacobson SM, Edwab RR, Pillar R. Ameloblastic fibro-odontoma. N Y State Dent J 1986;52:20-1.

51. Reich RH, Reichart PA, Ostertag H. Ameloblastic fibro-odontome. Report of a case, with ultrastructural study. J Maxillofac Surg 1984;12:230-4.

52. Anneroth G, Modéer T, Twetman S. Ameloblastic fibro-odontoma in the maxillae. A case report. Int J Oral Surg 1982;11:130-4.

53. Hutt PH. Ameloblastic fibro-odontoma: Report of a case with documented four-year follow-up. J Oral Maxillofac Surg 1982;40:45-8.

54. Daley TD, Lovas GL. Ameloblastic fibro-odontoma: Report of a case. J Can Dent Assoc 1982;48:467-9.

55. Curran JB, Owen D, Lanoway J. Ameloblastic fibro-odontoma-case report. J Can Dent Assoc 1980;46:314-6.

56. Bernhoft CH, Bang G, Gilhuus-Moe O. Ameloblastic fibro-odontoma. Int J Oral Surg 1979;8:241-4.

57. Geller LJ, Fielding AF. Ameloblastic fibro-odontoma. Report of case. Dist Columbia Dent Soc J 1978;65-70.

58. Howell RM, Burkes EJ Jr. Malignant transformation of ameloblastic fibro-odontoma to ameloblastic fibrosarcoma. Oral Surg Oral Med Oral Pathol 1977;43:391-401.

59. Album MM, Neff JH, Myerson RC. Ameloblastic fibro-odontoma: Report of case. ASDC J Dent Child 1977;44:320.

60. Herd JR. An ameloblastic fibro-odontome. Aust Dent J 1977;22:1-5.

61. Eda S, Tokuue S, Kato K, Uchida E, Yoshida T. A melanotic ameloblastic fibro-odontoma. Bull Tokyo Dent Coll 1977;18:119-28.

62. Cran JA, Herd JR, Chau KK. An unusual odontogenic lesion. Case report. Aust Dent J 1976;21:520-4.

63. Hanna RJ, Regezi JA, Hayward JR. Ameloblastic fibro-odontoma: Report of case with light and electron microscopic observations. J Oral Surg 1976;34:820-5
64. Miller AS, López CF, Pullon PA, Elzay RP. Ameloblastic fibro-odontoma. Report of seven cases. Oral Surg Oral Med Oral Pathol 1976;41:354-65.

65. Meerkotter VA. The ameloblastic fibro-odontome - Report of a case and the histogenesis of an anomalous denticle. J Dent Assoc S Afr 1974;29:389-93.

66. Sanders DW, Kolodny SC, Jacoby JK. Ameloblastic fibro-odontoma: Report of case. J Oral Surg 1974;32:281-5.

67. 67Worley RD, McKee PE. Ameloblastic odontoma: Report of case. J Ora Surg 1972;30:764-6.

68. O'Brien FV. Ameloblastic odontome. A case report. Br Dent J 1971;131:71-2.

69. Dutta A. Ameloblastic odontoma. Oral Surg Oral Med Oral Pathol 1970;29:827-31.

70. Jacobsohn PH, Quinn JH. Ameloblastic odontomas. Report of three cases. Oral Surg Oral Med Oral Pathol 1968;26:829-36.

71. Hamner JE $3^{\text {rd }}$, Pizer ME. Ameloblastic odontoma. Report of two cases. Am J Dis Child 1968;115:332-6.

72. Olech E, Alvares O. Ameloblastic odontoma. Report of a case. Oral Surg Oral Med Oral Pathol 1967;23:487-92.

73. Barnes L, Eveson JW, Reichart P, Sidransky D, editors. Pathology and Genetics of Head and Neck Tumors. Lyon: IARC Press; 2005. p. 286.

74. Kramer IR, Pindborg JJ, Shear M. Histological Typing of Odontogenic Tumours. Berlin: Springer-Verlag; 1992. p. 18.

75. Choukas NC, Toto PD. Ameloblastic odontoma. Oral Surg Oral Med Oral Pathol 1964;17:10-5.

76. Frissell CT, Shafer WG. Ameloblastic odontoma; report of a case. Oral Surg Oral Med Oral Pathol 1953;6:1129-33.

77. Herzog U, Putzke HP, Bienengräber V, Radke C. The ameloblastic fibro-odontoma - An odontogenic mixed tumor progressing into an odontogenic sarcoma. Dtsch Z Mund Kiefer Gesichtschir 1991;15:90-3.

78. Bregni RC, Taylor AM, García AM. Ameloblastic fibrosarcoma of the mandible: Report of two cases and review of the literature. J Oral Pathol Med 2001;30:316-20.

79. Gatz SA, Thway K, Mandeville H, Kerawala C, MacVicar D, Chisholm J Chemotherapy responsiveness in a patient with multiply relapsed ameloblastic fibro-odontosarcoma of the maxilla. Pediatr Blood Cancer 2015;62:2029-32. 